\title{
Entanglement generated by dissipation and steady state entanglement of two macroscopic objects
}

\author{
Hanna Krauter ${ }^{1 *}$, Christine A. Muschik ${ }^{2 *}$, Kasper Jensen ${ }^{1}$, Wojciech \\ Wasilewski $^{1, \dagger}$, Jonas M. Petersen ${ }^{1}$, J. Ignacio Cirac ${ }^{2}$, and Eugene S. Polzik ${ }^{1, \ddagger}$ \\ 1 Niels Bohr Institute, \\ Danish Quantum Optics Center QUANTOP, \\ Copenhagen University, Blegdamsvej 17, \\ 2100 Copenhagen Denmark. \\ ${ }^{2}$ Max-Planck-Institut für Quantenoptik, \\ Hans-Kopfermann-Strasse, D-85748 Garching, Germany \\ * These authors contributed equally to this work.
}

\begin{abstract}
Entanglement is a striking feature of quantum mechanics and an essential ingredient in most applications in quantum information. Typically, coupling of a system to an environment inhibits entanglement, particularly in macroscopic systems. Here we report on an experiment, where dissipation continuously generates entanglement between two macroscopic objects. This is achieved by engineering the dissipation using laser- and magnetic fields, and leads to robust event-ready entanglement maintained for $0.04 \mathrm{~s}$ at room temperature. Our system consists of two ensembles containing about $10^{12}$ atoms and separated by $0.5 \mathrm{~m}$ coupled to the environment composed of the vacuum modes of the electromagnetic field. By combining the dissipative mechanism with a continuous measurement, steady state entanglement is continuously generated and observed for up to an hour.
\end{abstract}

PACS numbers:

To date, experiments investigating quantum superpositions and entanglement are hampered by decoherence. Its effects have been studied in several systems [1. However, it was recognized [2] that the engineered interaction with a reservoir can drive the system into a desired steady state. In particular, dissipation common for two systems can drive them into an entangled state [3]. The idea of using and engineering dissipation rather than relying on coherent evolutions only, represents a paradigm shift with potentially significant practical advantages. Contrary to other methods, entanglement generation by dissipation does not require the preparation of a system in a particular input state and exists, in principle, for an arbitrary long time, which is expected to play an important role in quantum information protocols 4 4 . These features make dissipative methods inherently stable against weak random perturbations, with the dissipative dynamics stabilizing the entanglement.

We report on the first demonstration of purely dissipative entanglement generation [8]. In contrast to previous approaches 911 , entanglement is obtained without using measurements on the quantum state of the environment (i.e. the light field). The dissipation-based method implemented here is deterministic and unconditional and therefore fundamentally different from standard approaches such as the QND-based method [9] or the DLCZ protocol 4, which yield a separable state if the emitted photons are not detected. Furthermore, we report the creation of a steady state atomic entanglement by combining the dissipative mechanism proposed in [12] with continuous measurements. The generated entanglement is of the EPR type, which plays a central role in continuous variable quantum information processing [6, 13], quantum sensing [14] and metrology [11, 15, 16].

Fig. 1a presents the principles of engineered dissipation in our system consisting of two ${ }^{133} \mathrm{Cs}$ ensembles, interacting with a $y$-polarized laser field at $\omega_{L}$. A pair of twolevel systems is encoded in the $6 S_{1 / 2}$ ground state sublevels $|\uparrow\rangle_{I} \equiv|4,4\rangle_{I},|\downarrow\rangle_{I} \equiv|4,3\rangle_{I}$ and $|\uparrow\rangle_{I I} \equiv|4,-3\rangle_{I I}$, $|\downarrow\rangle_{I I} \equiv|4,-4\rangle_{I I}$. Operators $J_{\mathrm{I} / \mathrm{II}}^{ \pm}$with $J^{-}=\sum_{i=1}^{N}|\uparrow\rangle_{i}\langle\downarrow|$ describe collective spin flips, where $N$ is the number of atoms. The atoms are placed in a magnetic field in the $x$ direction and the collective operators $J_{y}=\sqrt{2}\left(J^{+}+J^{-}\right)$ and $J_{z}=i \sqrt{2}\left(J^{+}-J^{-}\right)$are defined in the frame rotating at the Larmor frequency $\Omega$. The two ensembles are initialized by optical pumping along the $x$-axis in the extreme states $m_{F}=4$ and $m_{F}=-4$ respectively, corresponding to $\left\langle J_{x}\right\rangle \equiv\left\langle J_{x, I}\right\rangle=-\left\langle J_{x, I I}\right\rangle \approx 4 N$ (see Fig. 1 ). Within the Holstein-Primakoff approximation, we introduce the canonical variables $X_{I / I I}=J_{y, I / I I} / \sqrt{\left|\left\langle J_{x}\right\rangle\right|}$ and $P_{I / I I}= \pm J_{z, I / I I} / \sqrt{\left|\left\langle J_{x}\right\rangle\right|}[6]$. The EPR entanglement condition [9, 17] for such ensembles is given by $\xi=$ $\Sigma_{J} /\left(2\left|\left\langle J_{x}\right\rangle\right|\right)=\operatorname{var}\left(X_{I}-X_{I I}\right) / 2+\operatorname{var}\left(P_{I}+P_{I I}\right) / 2<1$, where $\Sigma_{J}=\operatorname{var}\left(J_{y, \mathrm{I}}-J_{y, \mathrm{II}}\right)+\operatorname{var}\left(J_{\mathrm{z}, I}-J_{z, \mathrm{II}}\right)$. The entangling mechanism is due to the coupling to the $x$ polarized vacuum modes in the propagation direction $z$ of the laser field (Fig. 1), which are shared by both ensembles and provide the desired common environment. Spin flip processes in the two samples accompanied by forward scattering result in indistinguishable photons leading to quantum interference and entanglement of the ensembles. These spin flips and the corresponding photon scattering (see level schemes in Fig. 1) are described by the inter- 


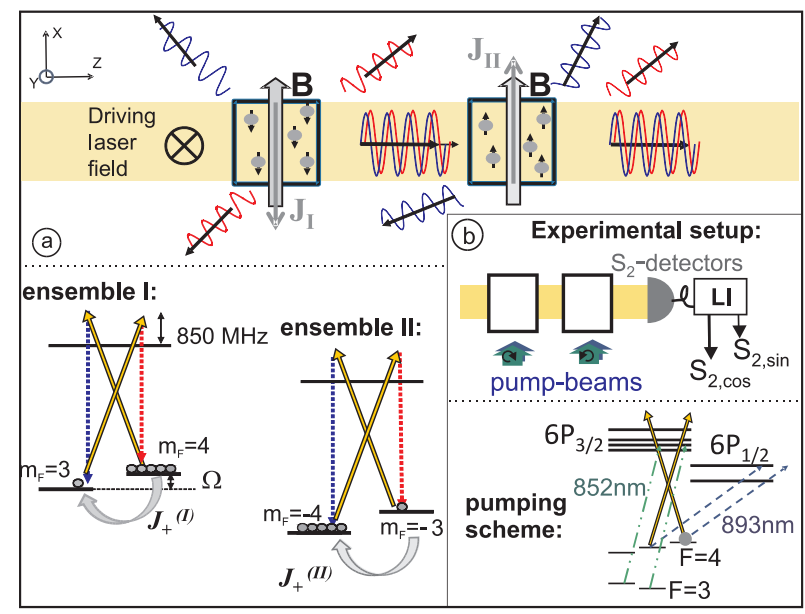

FIG. 1: a) Collective dissipation modes and atomic levels: two spatially separated atomic ensembles interact with the environment composed of the vacuum modes of the electromagnetic field. The coupling is driven by the $y$-polarized laser beam. The engineered collective dissipation is due to photons scattered in the forward $z$-direction. Internal level scheme of the atoms: the effective two-level systems $|\uparrow\rangle$ and $|\downarrow\rangle$ are two magnetic sublevels Zeeman-shifted by a magnetic field applied in the $x$-direction, which defines the quantization axis. Atoms in the two ensembles are initialized in opposite spin states. The laser beam off-resonantly couples these levels to the excited states and to the electromagnetic vacuum modes. Due to the Zeeman shift of the ground state levels, photons are emitted into the upper and lower sidebands (shown in blue and red color) leading to collective spin flips $J_{ \pm}$. b) Geometry of the experiment. The $S_{2}$ detector signal processed by the lock-in amplifier (LI) is used to determine the atomic quantum spin components $J_{y, z}$ as described in the text. The optical pumping scheme is also shown.

action Hamiltonian of the type

$H \propto \int_{\Delta \omega_{\mathrm{ls}}} d \mathbf{k}\left(A a_{\mathbf{k}}^{\dagger}+A^{\dagger} a_{\mathbf{k}}\right)+\int_{\Delta \omega_{\mathrm{us}}} d \mathbf{k}\left(B a_{\mathbf{k}}^{\dagger}+B^{\dagger} a_{\mathbf{k}}\right)$ where the integrals cover narrow bandwidths centered around the lower and upper sideband at $\omega_{L} \mp \Omega$ respectively and with the non-local spin operators $A=$ $\mu J_{\mathrm{I}}^{-}-\nu J_{\mathrm{II}}^{-}, B=\mu J_{\mathrm{II}}^{+}-\nu J_{\mathrm{I}}^{+}$. The fact that the electromagnetic modes $a_{\mathbf{k}}^{\dagger}$ form a continuum is crucial for the entanglement to be created without measurements [12]. As emission into the forward direction is collectively enhanced for a large optical depth $d[6$, the forward scattered modes can successfully compete with spontaneous emission modes in directions other than $z$ which leads to decoherence of the atomic state. Note that the generation of entanglement cannot be explained by the interaction of photons emitted by the first ensemble with the second one, which is negligible in our parameter regime. The nonlocal dissipative atomic dynamics obtained after tracing over the photonic modes is governed by the master equation [12:

$\frac{d}{d t} \rho=d \frac{\Gamma}{2}\left(A \rho A^{\dagger}-A^{\dagger} A \rho+B \rho B^{\dagger}-B^{\dagger} B \rho+H . C.\right)+$ $\mathcal{L}_{\text {noise }} \rho$, where $\rho$ is the atomic density operator, and $\Gamma$ is the single atom radiative decay. The Lindblad terms in parentheses, which would usually describe regular spontaneous emission, drive the system into an EPR state with $\xi=(\mu-\nu)^{2}<1$ [12, due to the special nonlocal construction of $A$ and $B . \mathcal{L}_{\text {noise }}$ describes undesired processes such as single atom spontaneous emission, collisions, etc. The experiments are performed using two dilute ${ }^{133} \mathrm{Cs}$ gas samples in $2.2 \mathrm{~cm}$ cubic cells separated by $0.5 \mathrm{~m}$ described elsewhere [6]. A bias magnetic field of $0.9 \mathrm{G}$ leads to a Zeeman splitting of $\Omega=2 \pi \cdot 322 \mathrm{kHz}$ (see Fig. 1). The anti-relaxation coating of the cell walls and careful magnetic shielding 14 provide the nonradiative decoherence time for populations and coherences of $T_{1} \approx 130 \mathrm{~ms}$ and $T_{2} \approx 40 \mathrm{~ms}$. The two ensembles are initialized in the states $|4, \pm 4\rangle$ with orientation up to $P=0.998$ (3) by applying a pump laser polarizing the $F=4$ manifold and a laser repumping atoms from $F=3$ to $F=4$ for 10 to $50 \mathrm{~ms}$ (Fig. 1b). The driving laser is blue detuned by $850 \mathrm{MHz}$ from the $F=4 \leftrightarrow F=5$ transition of the $D_{2}$ line corresponding to $(\mu-\nu)^{2}=0.16$. The laser power influences both the collective and the single atom dissipation processes and has been optimized within a range of $5-15 \mathrm{~mW}$. The nonlocal atomic state variance $\xi=\Sigma_{J} /\left(2\left|\left\langle J_{x}\right\rangle\right|\right)$ is inferred, and the entanglement condition $\xi<1$ is verified by a local polarization measurement on the light transmitted through the two ensembles (Fig. 1b). We use the same laser to create and to verify the entanglement which significantly simplifies the experiment. In the period $t<T$, up to a variable time $T$ (see the pulse sequence in Fig. 2b) the laser serves only as the driving source for dissipation. The results of the measurements on the transmitted light are not used, which is equivalent to tracing out the light field. Beginning at $t=T$, the temporal mode of the transmitted light is used for the determination of the atomic state at time $T$ using the established method [6, 11, 15, 18, of linear mapping of the atomic state onto light (atomic tomography via quantum polarization spectroscopy). The particular linear mapping used here has been utilized in several other contexts [14, 19, 20, and is described by the input-output relations for atomic and light operators before and after the interaction:

$$
\begin{aligned}
\frac{1}{\sqrt{2}}\left(X_{I}^{\text {out }}-X_{I I}^{\text {out }}\right) & =e^{-\gamma_{s} T} \cdot \frac{1}{\sqrt{2}}\left(X_{I}^{\text {in }}-X_{I I}^{\text {in }}\right)-\kappa(\mu-\nu)^{2} y_{c+}^{\text {in }}, \\
y_{c-}^{\text {out }} & =y_{c+}^{\text {in }} e^{-\gamma_{s} T}+\kappa \cdot \frac{1}{\sqrt{2}}\left(X_{I}^{\text {in }}-X_{I I}^{\text {in }}\right),
\end{aligned}
$$

and similarly for $X_{I}-X_{I I} \rightarrow P_{I}+P_{I I}$ and $y_{c} \rightarrow y_{s}$. Here $\kappa^{2}=\left(1-e^{-2 \gamma_{s} T}\right) /(\mu-\nu)^{2}$ and $\gamma_{s} \propto(\mu-\nu)^{2} J_{x} \Phi$, where $\Phi$ is the flux of photons in the drive field and $T$ is the interaction time. The light operators are given by the $\cos (\Omega t)$ and $\sin (\Omega t)$ components of the Stokes operator $S_{2}$ weighted with an exponentially falling (rising) mode function: $y_{c-/+}^{\text {out }(\text { in })}=\frac{1}{N_{ \pm} \sqrt{S_{x}}} \int_{0}^{T} S_{2}^{\text {out }(\text { in })}(t) \cos (\Omega t) e^{\mp \gamma_{s} t} d t$ (analogously for sine modes). Just as the master equation does, the input-output relations predict an entangled atomic state with variance $\Sigma_{J}(t) \rightarrow(\mu-\nu)^{2}$, for 


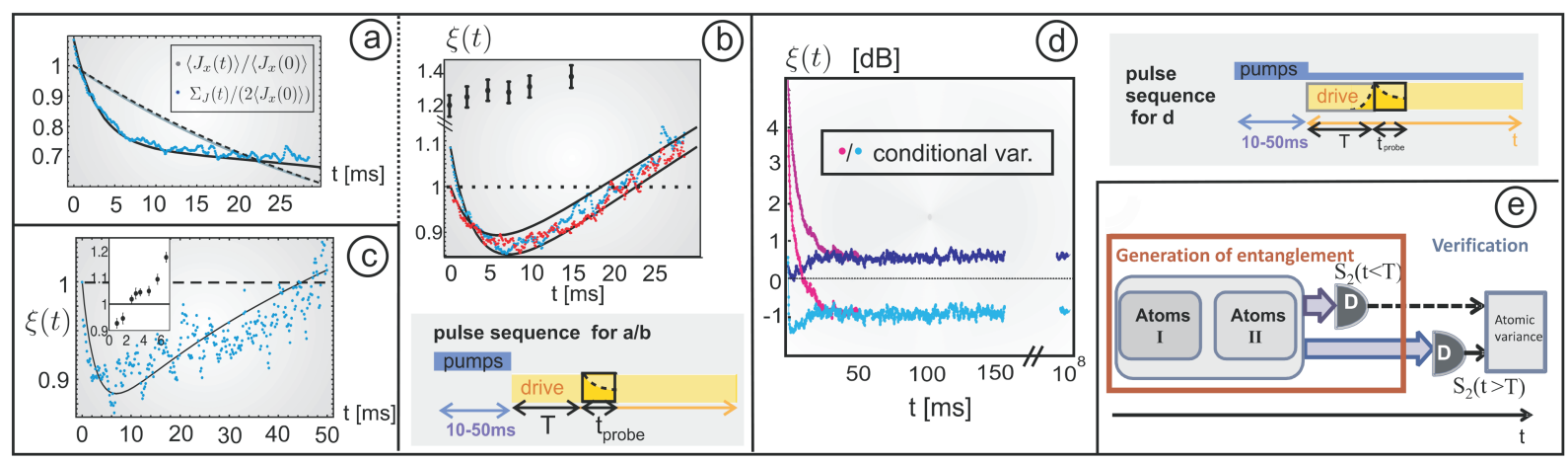

FIG. 2: Entanglement generated by dissipation (a-c) and steady state entanglement (d). a) Time evolution of $\Sigma_{J}(t) /\left(2\left|\left\langle J_{x}(0)\right\rangle\right|\right)$ (blue) and $\left\langle J_{x}(t)\right\rangle /\left\langle J_{x}(0)\right\rangle$ (grey). The theoretical fits (full and dashed black line) are based on the parameters $d=55$ (optical density), $\Gamma_{\text {col }}=0.002 \mathrm{~ms}^{-1}$ (collisional rate) and $\tilde{\Gamma}=0.193 \mathrm{~ms}^{-1}$ (dephasing rate [22]). The rates for driving field induced transitions $|4, \pm 3\rangle \rightarrow|4, \pm 4\rangle$ and $|4, \pm 4\rangle \rightarrow|4, \pm 3\rangle$ are given by $\mu^{2} \Gamma$ and $\nu^{2} \Gamma$ respectively, where $\Gamma=0.002 \mathrm{~ms}^{-1}$. b) Entanglement $\xi(t)$ versus time in ms. Blue data points correspond to the results shown in a). Data points in orange are obtained for a lower optical depth $(d=35)$. The other parameters used in the fits take the same values as in a). $\xi(t)<1$ certifies the creation of an inseparable state. The relevant pulse sequence is shown below. The data taken in the absence of the driving field (black points) show no entanglement. c) Dissipative entanglement generation in the presence of the pump field which incoherently transfers atomic population from undesired levels within $F=4$ back to the two level subsystem. The pump rate is $\Gamma_{\text {pump }}=0.168 \mathrm{~ms}^{-1} . d=37$, the fitting parameters $\Gamma_{\text {col }}$ and $\Gamma$ take the same values as in a) and $\tilde{\Gamma}=0.233 \mathrm{~ms}^{-1}$. The inset shows the evolution of $\xi(t)$ after the driving field is switched off after entanglement is generated by dissipation. d) Entanglement $\xi(t)$ for different initial conditions. The upper curves show a purely dissipative evolution. The lower curves the entanglement generated by dissipation combined with the measurement. Points on the right represent an average over measurements of one hour where atoms were kept in a steady state. The used exponential time mode functions are depicted in the pulse sequence and are described in the text. e) Schematic illustration of entanglement generation and verification. The signal from the detector $\mathrm{D}$ for times $t>T$ is used for verification of entanglement in (a-c). In $\mathrm{d})$ the signal taken at $t<T$ is given to the verifier as additional information.

$T>>\gamma_{s}^{-1}$. For $(\mu-\nu)^{2}<<1$ and finite $\gamma_{s} T$ the inputoutput relations reduce to the quantum-nondemolition type. The atomic EPR variance $\Sigma_{J}(T)$ at time $T$ can be inferred by using the input-output relations (1). The Stokes operator $S_{2}$ (the photon flux difference between $+45^{\circ}$ and $-45^{\circ}$ polarizations with respect to the $y$-axis in Fig. 1b) is measured in the time interval $\left[T ; T+t_{\text {probe }}\right]$ (see pulse sequence in Fig. 2b) with the photocurrent electronically processed to obtain the relevant light mode: $y_{c-}^{\text {read out }} \propto \int_{T}^{T+t_{\text {probe }}} S_{2}^{\text {out }}(t) \cos (\Omega t) e^{-\gamma_{s} t} d t$. The parameters $\kappa^{2}, \gamma_{s},(\mu-\nu)$ of the linear input-output relations are calibrated as described elsewhere [14,19]. The atomic state reconstruction is calibrated and verified carefully as described in detail in the supplemental material SM [21], where also the modification of the input output equations by losses and decoherence is presented. We conclude that the measurement of $\xi$ is reliable within the uncertainty of $\pm 4 \%$ arising from uncertainty in the measurements of $\kappa^{2}$, the detection efficiency $\eta$ and the shot noise of light.

In the first set of experiments, entanglement is generated purely dissipatively. In the first series of this set, the pump- and repump fields are turned off at time $t=0$ (Fig. 2a,b) and the driving (entangling) laser is turned on. In the presence of the drive field $(P \approx 5.6 \mathrm{~mW}) T_{2}$ is reduced to $6 \mathrm{~ms}$ and $T_{1}$ to $34 \mathrm{~ms}$. This decoherence has been considered the fundamental limitation for the entanglement generated by measurements [6]. Here, the collective entangling dissipation due to forward scattering dominates over the single atom decoherence and leads to a rapid reduction of $\Sigma_{J}(t)$ on the time scale of $\gamma_{s}^{-1}$. Fig. 2a shows the time evolution of $\Sigma_{J}(t)$ normalized to $2\left|\left\langle J_{x}(0)\right\rangle\right|$. For a Coherent Spin State (CSS) $\xi=1$, and $\Sigma_{\text {CSS }}=2\left|\left\langle J_{x}\right\rangle\right|$ defines the projection noise (PN) level, below which lies the noise level of entangled states. The dynamics of $2\left|\left\langle J_{x}(t)\right\rangle\right|$ due to single atom spontaneous emission and collisions on the slow time scale of $T_{1}$ is also shown in Fig. 2a. Fig. 2b presents the time evolution of entanglement for two values of the optical depth $d \approx 34\left(\Theta=8.5^{\circ}\right)$ and $d \approx 56\left(\Theta=14.0^{\circ}\right)$. The data is well fitted with theory [12] using the collisional rate $\Gamma_{\text {col }}$ and dephasing rate $\tilde{\Gamma}[22$ compatible with experimental values. The details of calculations of the fits are given in 21. The time interval 0.015 s over which entanglement is continuously maintained is several times longer than the best previous results obtained for measurement induced entanglement [5, 6] and much longer than $T_{2}$. For comparison, if the driving (entangling) laser is off during $0<t<T$ and is turned on only at $t=T$ to measure the atomic state, $\xi(T)$ predictably stays above the PN level (black points in Fig. 2b). Also a slight mismatch of the Larmor frequencies of the two ensembles in the preparation period by $\sim 20 \mathrm{~Hz}$ leads to the disappearance of the entanglement. This can be viewed as a direct consequence of the "which way" information due 
to the distinguishability of photons emitted by the two ensembles.

In the series presented above, entanglement is created in a quasi steady state rather than in a steady state, as would be the case for atoms with a true two-level atomic ground state, for example in Ytterbium ensembles [23]. On the time scale of $T_{1}$, atoms are lost to other magnetic sublevels of $F=4$ and to the level $F=3$. This causes the eventual extinction of entanglement as described well by the theoretical fits shown in Fig. 2a,b with the pumping rate $\Gamma_{\text {pump }}$ being close to the experimental value. In the next series of experiments, the pumping field of an optimal strength resonant with the $F=4$ state is kept on during the entanglement generation period $t>0$ ( Fig. 2c). Remarkably, this incoherent process does not suppress generation of entanglement, but on the contrary brings it further towards a steady state. The entanglement can now be maintained for 0.04 s thanks to pumping atoms from sublevels $\left|m_{F}\right| \leq 3$ which contribute higher noise, back to $\left|m_{F}\right|=4$ which is a dark state for the pump beam. The eventual loss of entanglement, is in part due to atoms which are lost to the $F=3$ ground state, effectively reducing $d$. If the entangling mechanism is turned off, the entangled state decays in $2 \mathrm{~ms}$ (inset in Fig. 2c), as expected [6] from the decoherence in the dark.

Finally, we demonstrate generation of steady state atomic entanglement. To this end, a repumping field $F=3 \rightarrow F=4$ is added during the entanglement generation, thus closing down the last escape channel from the relevant spin system. The atoms reach a steady state which is, however, not entangled because the collective processes are not sufficiently strong to overcome the noise added by the incoherent repumping field. Theory predicts 12 that a steady state entanglement can be achieved for $d=100$, but this is experimentally unfeasible. However, we can use the fact that due to single atom decoherence sources, the atomic state is not pure, and hence forward scattered light is not completely disentangled from the atoms. Up to now, measurements on light variables have only been used to verify entanglement at time $\mathrm{T}$ for which only $y_{c, s-}^{\text {out }}(t>T)$ have been utilized. Using the results of the continuous measurement on the open atomic quantum system during the interval $t<T$, we can enhance the entanglement generated by dissipation and maintain it in the steady state. In this scenario, the verifier (Fig. 2e) receives the classical information $S_{2}^{\text {out }}(t<T)$ which is used to calculate the conditional variance $\operatorname{var}\left(y_{c, s}^{\text {cond }}\right)=\operatorname{var}\left(y_{c, s}^{\text {read out }}(T)-\alpha y_{c, s}^{\text {feed }}(t<T)\right)$. Here, $y_{c}^{\text {feed }}(t<T)=\frac{1}{N_{f} \sqrt{S_{x}}} \int_{0}^{T} S_{2}^{\text {out }}(t) \cos (\Omega t) e^{\gamma_{m} t} d t$ and the feedback gain $\alpha$ and the time constant $\gamma_{m}$ are optimized to achieve maximal noise reduction. The light mode that brings about the best noise reduction is a fast growing exponential mode, with $\gamma_{m}=0.83 \mathrm{~ms}^{-1}>$ $\gamma=1 / T_{2}=0.27 \mathrm{~ms}^{-1}$. The conditionally reduced atomic variance $\xi_{\text {cond }}=\operatorname{var}\left[\frac{1}{2}\left(X_{I}-X_{I I}\right)+\frac{1}{2}\left(P_{I}-P_{I I}\right)-\frac{\alpha}{\kappa}\left(y_{c}^{\text {feed }}+\right.\right.$ $\left.\left.y_{s}^{\text {feed }}\right)(t<T)\right)$ ] is then found from $\operatorname{var}\left(y_{c, s}^{\text {cond }}\right)$ using the same calibrated input-output relations as above. These central results are displayed in Fig. 2d, which shows the evolution of the variances of the purely dissipatively generated atomic state (upper curves) and the entanglement produced using the hybrid method including dissipation and continuous measurements (lower curves). Each pair of curves is taken with two different initial conditions. These results demonstrate a very important aspect of our work, - they show that the generated steady state is independent of the initial state, and that entanglement is maintained for up to an hour, if dissipative processes are combined with measurements.

In conclusion, we have observed entanglement of macroscopic atomic ensembles generated by dissipation and the steady state atomic entanglement. The results present a new step in quantum control of entanglement. Dissipatively generated entanglement provides not only event-ready entangled links for standard protocols but is also an elementary resource for future applications in continuous quantum information processing schemes, such as dissipative distillation and repeater protocols, which allow for the distribution of long-range high-quality steady state entanglement [7.

\section{Acknowledgements}

We acknowledge support from the Elite Network of Bavaria (ENB) project QCCC and the EU projects COMPAS, Q-ESSENCE and QUEVADIS. C.A.M. acknowledges valuable discussions with K. Hammerer, K.G.H. Vollbrecht and G. Giedke.

\section{Author Contributions}

H.K. and K.J. obtained the experimental data with the assistance of J.M.P. W.W. performed preliminary measurements. C.M. developed the theory. J.I.C. and E.S.P. planned and supervised the work.

$\dagger$ Current address: Institute of Experimental Physics, University of Warsaw, Hoza 69, PL00681 Warsaw, Poland

‡ Author for correspondence. E-mail: polzik@nbi.dk

[1] C. Myatt et al., Nature 403, 269 (2000); S. Gleyzes et al., Nature 446, 297 (2007); S. Hofferberth et al., Nature 449, 324 (2007); M. Branderhorst, et al., Science 320, 638 (2008); N. Syassen et al., Science 3201329 (2008); J. Barreiro et al., Nature 470, 486 (2011); R. Bloomer, M. Pysher, and O. Pfister, New J. Phys. 13063014 (2011).

[2] J. F. Poyatos et al., Phys. Rev. Lett. 77, 4728 (1996). 
[3] M. B. Plenio and S. F. Huelga, Phys. Rev. Lett. 88, 197901 (2002); B. Kraus and J. I. Cirac, Phys. Rev. Lett. 92, 013602 (2004); S. Diehl et al., Nature Phys. 4, 878 (2008); F. Verstraete, M. M. Wolf, and J. I. Cirac, Nature Phys. 5, 633 (2009); J. T. Barreiro, et al., Nature 470, 486 (2011). A. S. Parkins, E. Solano, and J. I. Cirac, Phys. Rev. Lett. 96, 053602 (2006).

[4] L.-M. Duan et al., Nature 414, 413 (2001).

[5] H. J. Kimble, Nature 453, 1023 (2008).

[6] K. Hammerer, A. S. Sørensen, and E. S. Polzik, Rev. Mod. Phys. 82, 1041 (2010).

[7] K. G. H. Vollbrecht, C. A. Muschik, and J. I. Cirac, arXiv:1011.4115 (2010).

[8] First results demonstrating the creation of entanglement by dissipation are reportet in the unpublished paper: $\mathrm{H}$. Krauter, C. A. Muschik, K. Jensen, W. Wasilewski, J. M. Petersen, J. I. Cirac, and E. S. Polzik, arXiv:1006.4344 (2010).

[9] B. Julsgaard, A. Kozhekin, and E. S. Polzik, Nature 413, 400 (2001).

[10] D. N. Matsukevich et al., Phys. Rev. Lett. 96, 030405 (2006); C. W. Chou et al., Nature 438, 828 (2005); M. Eisaman et al., Nature 438, 837 (2005); Z. Yuan et al., Nature 454, 1098 (2008).

[11] J. Appel et al., PNAS 106, 10960 (2009).

[12] C. A. Muschik, E. S. Polzik, and I. J. Cirac, Phys. Rev. A 83, 052312 (2011).

[13] A. Furusawa and N. Takei, Phys. Rep. 443, 97 (2007).

[14] W. Wasilewski et al., Phys. Rev. Lett. 104, 133601 (2010).

[15] M. H. Schleier-Smith, I. D. Leroux, and V. Vuletić, Phys. Rev. Lett. 104, 073604 (2010).

[16] C. Gross et al., Nature 464, 1165 (2010); M. F. Riedel et al., Nature 464, 1170 (2010).

[17] L.-M. Duan et al., Phys. Rev. Lett. 84, 2722 (2000).

[18] M. Koschorreck et al., Phys. Rev. Lett. 105, 093602, (2010).

[19] W. Wasilewski et al., Opt. Express 17, 144444 (2009).

[20] K. Jensen et al., Nature Phys. 7, 13 (2011).

[21] Supplemental material.

[22] The effective dephasing rate associated with noise effects $\tilde{\Gamma}=\Gamma_{\text {cool }}+\Gamma_{\text {heat }}+\Gamma_{\text {deph }}$ includes the effective single particle cooling rate $\Gamma_{\text {cool }}$ (which is a sum of rates corresponding to cooling processes due to the driving and pump field and collisions), the effective single particle heating rate $\Gamma_{\text {heat }}=\Gamma_{\uparrow \downarrow}=\nu^{2} \Gamma+\gamma_{\text {col }}$ as well as pure dephasing $\Gamma_{\text {deph }}$.

[23] T. Takano et al., Phys. Rev. Lett. 102, 033601 (2009).

\section{SUPPLEMENTAL MATERIAL}

The first part of the supplemental material contains details of the calibration of the atomic spin noise with respect to the projection noise and of the orientation of the collective atomic spin.

The second part contains details of the theoretical fits presented in Fig. 2 of the main text.

\section{Projection noise calibration}

The variances of the collective atomic operators $P_{I}^{\mathrm{in}}+P_{I I}^{\mathrm{in}}$ and $X_{I}^{\mathrm{in}}-X_{I I}^{\mathrm{in}}$ are found from the measured polarization mode of the transmitted light $y_{c, s-}$ using the input-output relations given in Eq. (1) in the main text, as follows:

$\operatorname{var}\left(P_{I}^{\text {in }}+P_{I I}^{\text {in }}\right) / 2=\left(\operatorname{var}\left(y_{c-}^{\text {out }}\right)-\sigma_{\text {in }}^{2}\left(1-\kappa^{2}(\mu-\nu)^{2}\right)\right) / \kappa^{2}$, $\operatorname{var}\left(X_{I}^{\mathrm{in}}-X_{I I}^{\mathrm{in}}\right) / 2=\left(\operatorname{var}\left(y_{s-}^{\text {out }}\right)-\sigma_{\text {in }}^{2}\left(1-\kappa^{2}(\mu-\nu)^{2}\right)\right) / \kappa^{2}(\mathrm{~S} .1)$

where $\sigma_{i n}^{2}$ is the shot noise of light. The normalized EPR variance of atomic noise is $\xi=\operatorname{var}\left(P_{I}^{\mathrm{in}}+P_{I I}^{\mathrm{in}}\right) / 2+\operatorname{var}\left(X_{I}^{\mathrm{in}}-\right.$ $\left.X_{I I}^{\text {in }}\right) / 2$.

Decay of the atomic state with the rate $\gamma_{\text {extra }}$ can be included in the input-output equations (Eq. (1)) as follows:

$$
\begin{aligned}
\left(P_{I}^{\text {out }}+P_{I I}^{\text {out }}\right) / \sqrt{2}= & \left(P_{I}^{\text {in }}+P_{I I}^{\text {in }}\right) / \sqrt{2} e^{-\gamma T}-(\mu-\nu)^{2} \kappa y_{c+}^{\text {in }} \\
& +\epsilon \sqrt{1-e^{-2 \gamma T}} \cdot F_{p+} \\
\left(X_{I}^{\text {out }}-X_{I I}^{\text {out }}\right) / \sqrt{2}= & \left(X_{I}^{\text {in }}-X_{I I}^{\text {in }}\right) / \sqrt{2} e^{-\gamma T}-(\mu-\nu)^{2} \kappa y_{s+}^{\text {in }} \\
& +\epsilon \sqrt{1-e^{-2 \gamma T}} \cdot F_{x-}
\end{aligned}
$$

with the total decay $\gamma=1 / T_{2}=\gamma_{s}+\gamma_{\text {extra }}, \epsilon^{2}=\gamma_{\text {extra }} / \gamma$ S1 and the coupling constant $\kappa=\sqrt{\left(1-\epsilon^{2}\right)\left(1-e^{-2 \gamma T}\right)} /(\mu-\nu)$. The two-cell noise operators $\left\langle F_{i}^{2}\right\rangle=\frac{1}{2}$ model a decay towards the coherent spin state (CSS), which is a good approximation on short interaction timescales. The equations for light are adjusted accordingly and used for the reconstruction of the atomic noise. The coupling constant $\kappa^{2}$ is calibrated as discussed in [14]. In Fig. S.1A, measurements of the coupling constant are shown for different atom numbers, monitored by the Faraday angle $\Theta \propto J_{x}$ which is measured by the polarization rotation of an independent probe beam propagating in $x$-direction (see Fig. S.2). $(\mu-\nu)^{2}$ depends on detuning and has been found experimentally [19] and derived from the theory - both methods give the value $0.16 \pm 0.005$.

The imperfect detection efficiency $\eta=0.84(3)$ is included in the input-output equations via a simple beam splitter model. The light losses in the detection path which are relevant for the atomic noise reconstruction have been determined in two independent ways. They were measured directly and also inferred from the atomic noise as a function of the atom number presented in Fig. S.1p. In order to establish the projection noise $(\mathrm{PN})$ level we run a series of experiments in which we determine $\xi(t=0)$ (more precisely at $t=0.02 \mathrm{msec}$ ), i.e., right after the optical pumping is complete as a function of the macroscopic spin $J_{x}$. The number of atoms (macroscopic spins $J_{x}$ ) is varied by changing the temperature of the cells. We use a $1 \mathrm{~ms}$ drive pulse and perform measurements of $S_{2}$ as sketched in Fig. S.2. We then reconstruct the atomic noise with the equations discussed above. 


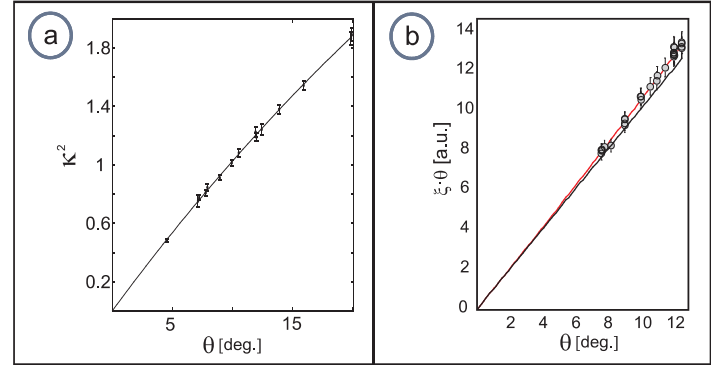

Supplemental figure S.1: Calibration measurements: a) Normalized initial spin noise variance as a function of $\Theta \propto$ $J_{x}$. The light power was held constant at $5.6 \mathrm{~mW}$, the pulse duration was $1 \mathrm{~ms}$. Red line - linear plus quadratic fit. Black line - linear part of the fit presenting the projection noise. b) Measurement results for $\kappa^{2}$ for different Faraday angles $\theta$.

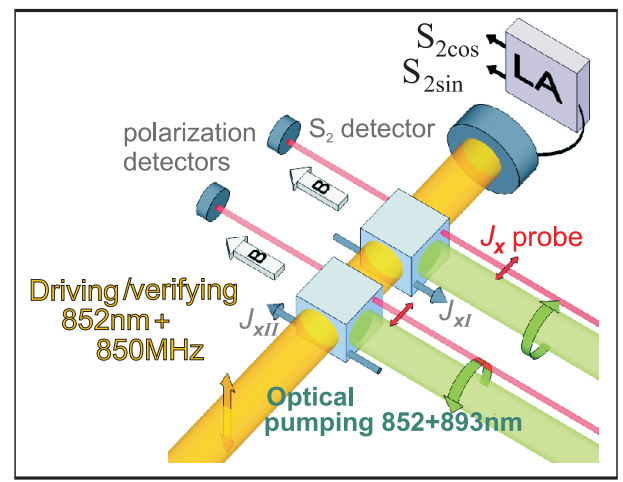

Supplemental figure S.2: Measurement setup and geometry of the experiment: The dc polarization detectors measure the Faraday rotation angle $\Theta$ proportional to the macroscopic spin $J_{x}$. The ac $S_{2}$ detector signal processed by the lock-in amplifier (LA) is used to determine the atomic quantum spin components $J_{y, z}$ as described in the main text.

We also independently measure the degree of spin polarization, as described below, exceeding 0.992 for all relevant atomic numbers. The results of the atomic noise measurements (Fig. S.1p) are well fit by $\xi \propto \Theta+0.004(3) \Theta^{2}$. This proves that the quadratic deviation from the $\mathrm{PN}$ is small and well characterized. For the presented range of number of atoms corresponding to $\Theta=6-11$ degrees, $\xi(0)$ is a few per cent above the PN-level. We also verify that $\xi$ is independent of the duration of the verifying measurement $t_{\text {probe }}$ for $0.5 \mathrm{~ms}<t_{\text {probe }}<3 \mathrm{~ms}$. We conclude that the measurement of $\xi$ is reliable within the uncertainty of $\pm 4 \%$ arising from uncertainty in the measurements of $\kappa^{2}$, the detection efficiency $\eta$, and the shot noise of light.

\section{Measurement of the macroscopic spin orientation}

The optimization of the orientation $o=\frac{1}{4} \sum_{m} m \cdot p_{m}$, where $p_{m}$ is the population of the magnetic sub-level $m$, is crucial for the success of the entanglement generation. Significant imperfections of the orientation lead to atomic noise above the PN

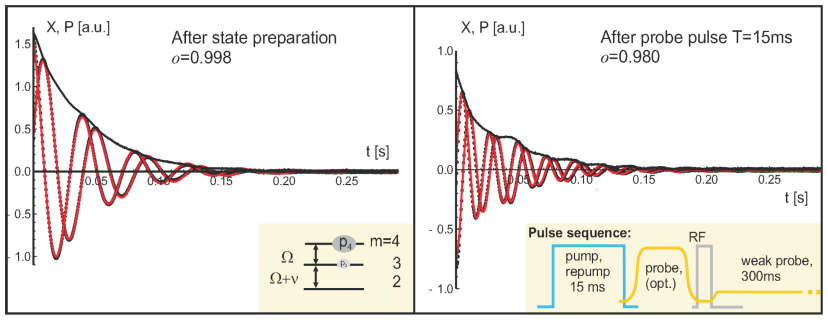

Supplemental figure S.3: Orientation test for the initial atomic state and after $15 \mathrm{~ms}$ probing with $5.6 \mathrm{~mW}$. The time evolution of a displacement in the two spin components in the rotating frame $X \propto\left\langle J_{y}\right\rangle$ and $P \propto\left\langle J_{z}\right\rangle$ is detected. The black dots are the measurement outcomes and the red lines the fitted curves, from which the orientation $o$ can be determined. The insets show the level structure (with $\nu \approx 20 \mathrm{~Hz}$ ) and the pulse sequence. The first probe pulse is optional.

level. Also several assumptions in the theoretical model are only valid for a highly oriented state. In the experiment the orientation was monitored as a function of time during the entire probe duration using a method closely related to the one described in [S2]. After the preparation of the CSS by optical pumping, we use a weak RF magnetic pulse at the Larmor frequency to create excitations causing a displacement of the rotating collective spin. This displacement is read out with a weak probe beam. The coherences between the different magnetic sub-levels oscillate at slightly different frequencies due to the second order Zeeman shift. In our case this splitting is $20 \mathrm{~Hz}$. When we look at the evolution of the displacement demodulated with an RF frequency, close to the Larmor frequency $\Omega$, a population of sub-levels other than $m=4$ will manifest itself in a quantum beat signal at multiples of the second order Zeeman splitting frequency. In Fig. S.3. two signals for slightly different orientations are shown and the difference is clearly visible. This method is remarkably sensitive to orientation imperfections. After the pumping, we start very close to the CSS with $o=0.998(3)$. After a $15 \mathrm{~ms}$ probe pulse with $5.6 \mathrm{~mW}$ the orientation is reduced to $o=0.980(3)$.

\section{Comparison of experimental data and theoretical predictions}

The generated amount of entanglement $\xi(t)$ can be calculated as described in detail in [12]. As explained there, the produced entanglement is given by

$$
\xi(t)=\frac{\Sigma_{J}(t)+14 N_{|4, \pm 3\rangle}(t)}{N_{2}(t)\left(P_{2}(t)+7\right)}
$$

where $N_{|4, \pm 3\rangle}\left(N_{\uparrow / \downarrow}\right)$ is the number of particles in state $|4, \pm 3\rangle(|\uparrow\rangle /|\downarrow\rangle)$ and $N_{2}=N_{\uparrow}+N_{\downarrow}$ and $P_{2}=\left|N_{\uparrow}-N_{\downarrow}\right| / N_{2}$ denote the particle number and orientation with respect to the two-level subsystem respectively. The time evolution of the EPR variance $\Sigma_{J}=\operatorname{var}\left(J_{y, I}-J_{y, I I}\right)+\operatorname{var}\left(J_{z, I}-J_{z, I I}\right)$ (see main text) can be calculated using Eq. (21) in [12] if the collective decay rate $(d \Gamma)$ and the effective dephasing rate $(\tilde{\Gamma})$ as well as $N_{2}(t)$ and $P_{2}(t)$ are known. In the following we describe how these quantities can be inferred and explain the 
theoretical fits to the measured data presented in the main text.

The essential features of the experiment can be described by means of a simplified model, which allows one to take additional dynamics due to the multilevel character of Cesium into account and involves only the three atomic states $|4, \pm 4\rangle$, $|4, \pm 3\rangle$ and $|h\rangle_{I / I I} \equiv|3, \pm 3\rangle$. For the timescales considered here, atomic population in other sublevels can be neglected. Since the model is primarily intended to describe qualitatively the physical effects observed in the experiment with very few parameters, we use $\Gamma_{|4, \pm 4\rangle \rightarrow|h\rangle} \approx \Gamma_{|4, \pm 3\rangle \rightarrow|h\rangle}=\Gamma_{\text {out }}$ and $\Gamma_{|h\rangle \rightarrow|4, \pm 4\rangle} \approx \Gamma_{|h\rangle \rightarrow|4, \pm 3\rangle}=\Gamma_{\text {in }}$ such that

$$
\begin{aligned}
\frac{d}{d t} N_{2}(t)= & -\left(\Gamma_{\text {out }}+2 \Gamma_{\text {in }}\right) N_{2}(t)+2 N \Gamma_{\text {in }}, \\
\frac{d}{d t} \tilde{P}_{2}(t)= & -\left(\Gamma_{3,4}+\Gamma_{4,3}+\Gamma_{\text {out }}\right) \tilde{P}_{2}(t) \\
& +\left(\Gamma_{3,4}-\Gamma_{4,3}\right) N_{2}(t) / N,
\end{aligned}
$$

where $\tilde{P}_{2}(t)=P_{2}(t) N_{2}(t) / N$. Here and in the following we use the abbreviations $\Gamma_{|4, \pm 4\rangle \rightarrow|4, \pm 3\rangle}=\Gamma_{4,3}$ and $\Gamma_{|4, \pm 3\rangle \rightarrow|3, \pm 4\rangle}=$ $\Gamma_{3,4}$. Atomic transitions can be either induced by the driving field or due to collisions. Since the thermal energy of atoms is much larger than the atomic level splittings, we assume the same collisional rate $\Gamma_{\text {col }}$ for all atomic transitions. Accordingly,

$$
\begin{aligned}
\Gamma_{3,4} & =\mu^{2} \Gamma+\Gamma_{\mathrm{col}}, & \Gamma_{\mathrm{out}} & =\Gamma_{\mathrm{L}}^{\mathrm{out}}+\Gamma_{\mathrm{col}}, \\
\Gamma_{4,3} & =\nu^{2} \Gamma+\Gamma_{\mathrm{col}}, & \Gamma_{\mathrm{in}} & =\Gamma_{\mathrm{col}},
\end{aligned}
$$

where the abbreviations $\Gamma_{|4, \pm 4\rangle \rightarrow|4, \pm 3\rangle}=\Gamma_{4,3}$ and $\Gamma_{|4, \pm 3\rangle \rightarrow|3, \pm 4\rangle}=\Gamma_{3,4}$ have been used. $\mu^{2} \Gamma$ and $\nu^{2} \Gamma$ are the driving field induced cooling and heating rate respectively. $\Gamma_{\mathrm{L}}^{\text {out }}$ is the rate at which atoms leave the two level subsystem due to radiative transitions caused by the driving field. The number of free parameters in these equations can be reduced to two, using the experimentally determined time derivative of the atomic polarization $P=\left\langle J_{x}(t)\right\rangle /\left\langle J_{x}(0)\right\rangle$ at time $t=0$

$\left.\frac{d}{d t} P(t)\right|_{t=0}=\frac{-\left(\Gamma_{4,3}+4 \Gamma_{\text {out }}\right) N_{|4, \pm 4\rangle}(0)+\left(\Gamma_{3,4}-3 \Gamma_{\text {out }}\right) N_{|4, \pm 3\rangle}(0)}{\left\langle J_{x}(0)\right\rangle}$,

where it is taken into account that the initial spin state is not perfectly polarized, but contains a small fraction of atoms in state $|4, \pm 3\rangle$. The initial populations $N_{|4, \pm 4\rangle}(0)=0.99$, $N_{|4, \pm 3\rangle}(0)=0.01$ and $N_{\mathrm{h}}(0)=0$ are estimated based on measurements of the orientation of the initial spin state after optical pumping which are described in the first part of SM. Using this constraint, $P(t)$ can be fitted with two free parameters. This way, fixed expressions for $P_{2}(t)$ and $N_{2}(t)$ are obtained. As mentioned above, the values of the collective decay rate $d \Gamma$ and the dephasing rate due to noise effects $\tilde{\Gamma}$ [S3] have to be known in order to calculate the generated amount of entanglement as described in [12]. These parameters are determined from the experimentally obtained slope of the variance $\Sigma_{J}(t) /\left(2\left|\left\langle J_{x}\right\rangle\right|\right)$ at time $t=0$

$$
\begin{aligned}
\left.\frac{d}{d t} \frac{\Sigma_{J}(t)}{2\left|\left\langle J_{x}(0)\right\rangle\right|}\right|_{t=0}=- & 4 N d \Gamma P_{2}(0)\left(1-P_{2}(0) /(\mu-\nu)^{2}\right) \\
& +7 \Gamma_{\text {in }} P_{2}(0)-7\left(\Gamma_{\text {out }}+\Gamma_{3,4}-\Gamma_{4,3}\right) N_{|4, \pm 3\rangle}(0),
\end{aligned}
$$

and the decay of the transverse spin $\left\langle J_{y}(t)\right\rangle=$ $e^{-\frac{1}{2}\left(\tilde{\Gamma}+d \Gamma \tilde{P}_{2}(t)\right) t}\left\langle J_{y}(0)\right\rangle$, where Eq. (21) in [12] and the identities relating quantities defined with respect to a two-level system to quantities defined with respect to a multi-level structure presented in Sec. IV.A in [12] have been used.

[S1] In the actual experiment, the $\gamma_{\text {extra }}$ will collect all decay mechanisms coming from spontaneous emission, collisions and magnetic field inhomogeneity

[S2] B. Julsgaard, J. Sherson, J. Sørensen, and E. S. Polzik, J. Opt. B, 6, 5 (2004).

[S3] In the absence of pump or repump fields, the dephasing rate associated with noise effects $\tilde{\Gamma}=\Gamma_{3,4}+\Gamma_{4,3}+\Gamma_{\text {deph }}$ includes the effective single particle cooling rate $\Gamma_{3,4}=$ $\mu^{2} \Gamma+\Gamma_{\text {col }}$, which is a sum of the rates corresponding to cooling processes due to the driving field and collisions, the effective single particle heating rate $\Gamma_{4,3}=\nu^{2} \Gamma+\Gamma_{\text {col }}$ as well as pure dephasing $\Gamma_{\text {deph }}$. 